\title{
On the solutions of a class of iterated generalized Bers-Vekua equations in Clifford analysis
}

\author{
P. Berglez
}

Department of Mathematics, Graz University of Technology, A-8010 Graz, Austria

\begin{abstract}
We consider functions with values in the Clifford algebra $C l_{p, q}$ which are solutions of a certain class of the iterated generalized Bers-Vekua equation $D^{m} w=0$ with $D w=\partial w+c \bar{w}$ where $\partial=\sum_{j=0}^{n} e_{j} \partial / \partial x_{j}$ is the generalized Cauchy-Riemann operator. We prove that any such function $w$ has a Almasi-type decomposition of the form $w=v_{0}+x_{0} v_{1}+\ldots+x_{0}^{m-1} v_{m-1}$ where $x=x_{0}+x_{1} e_{1}+\ldots x_{n} e_{n}$, and the functions $v_{j}, j=0,1, \ldots, m-1$, are solutions of the generalized Bers-Vekua equation $D v=0$.
\end{abstract}

Key words: iterated generalized Cauchy-Riemann operator; iterated generalized BersVekua operator; polymonogenic functions; differential operators of Bauer-type

\section{Introduction}

Let $C l_{p, q}$ with $p+q=n$ be the $2^{n}$-dimensional Clifford algebra generated by the elements $e_{1}, \ldots, e_{n}$, which obey the multiplication rules

$$
e_{j}^{2}=e_{0}, j=1, \ldots, p, e_{j}^{2}=-e_{0}, j=p+1, \ldots, n \quad \text { and } \quad e_{i} e_{j}+e_{j} e_{i}=0 \text { for } i<j,
$$

where $e_{0}$ denotes the identity of the algebra. An arbitrary element $a$ of $C l_{p, q}$ is given by

$$
a=\sum_{A} \lambda_{A} e_{A}, \lambda_{A} \in \mathbb{R}
$$

with $e_{A}=e_{\alpha_{1}} e_{\alpha_{2}} \ldots e_{\alpha_{k}}, \alpha_{1}, \ldots, \alpha_{k} \in\{1, \ldots, n\}$ and $1 \leq \alpha_{1}<\alpha_{2}<\ldots<\alpha_{k} \leq n$.

The conjugation is defined by $\bar{a}=\sum_{A} \lambda_{A} \bar{e}_{A}$ where $\bar{e}_{A}=\bar{e}_{\alpha_{k}} \ldots \bar{e}_{\alpha_{1}}$ and $\bar{e}_{0}=e_{0}$, $\bar{e}_{j}=-e_{j}, j=1, \ldots, n$, holds.

Now an element $x$ in $\mathbb{R}^{n+1}$ with the coordinates $x_{0}, x_{1}, \ldots, x_{n}$ is identified with

$$
x=x_{0}+x_{1} e_{1}+\ldots+x_{n} e_{n}
$$

and the conjugate of $x$ is $\bar{x}=x_{0}-x_{1} e_{1}-\ldots-x_{n} e_{n}$. 
In $\mathbb{R}^{n+1}$ the generalized Cauchy-Riemann operator is defined by

$$
\partial=\frac{\partial}{\partial x_{0}}+\sum_{j=1}^{n} e_{j} \frac{\partial}{\partial x_{j}}
$$

and the conjugate operator $\bar{\partial}$ is given by

$$
\bar{\partial}=\frac{\partial}{\partial x_{0}}-\sum_{j=1}^{n} e_{j} \frac{\partial}{\partial x_{j}}
$$

These operators act on the space $C^{1}\left(\Omega, C l_{p, q}\right)$ where $\Omega$ denotes a domain in $\mathbb{R}^{n+1}$.

Using such a Clifford algebra in a multidimensional space, elliptic, hyperbolic and parabolic differential equations can be considered in an inductive way (cf. e.g. [1]).

A function $u$ is said to be (left) Clifford holomorphic in $\Omega$ if it is a solution of the differential equation

$$
\partial u=0
$$

Here we consider the generalized Bers-Vekua operator $D$ defined by

$$
D v:=\partial v+c \bar{v}
$$

where $c$ is a real valued function of $x_{0}$.

In the algebra of complex quaternions $\mathrm{H}$. Malonek [2] investigated some classes of generalized Bers-Vekua equations. B. Goldschmidt [3] presented regularity properties of generalized analytic vectors a particular subset of which is described by $D v=0$. In [4] a similar operator was used to describe pseudoanalytic functions in the space, whereas in a forthcoming paper of the author for $c\left(x_{0}\right)=k / x_{0}, k \in \mathbb{Z}$, a representation theorem for the solutions of $D v=0$ is proved using certain differential operators of Bauer-type acting on Clifford holomorphic functions.

Here we study the iterated operator $D^{m}$ with $m \in \mathbb{N}$ and $D^{m} f=D\left(D^{m-1} f\right)$ and $D^{0} f=f$. There exists a close connection between the solutions of the iterated generalized Bers-Vekua equation

$$
D^{m} w=0
$$

and the solutions of $D v=0$. This result generalizes the representation of the solutions of the iterated generalized Cauchy-Riemann equation

$$
\partial^{m} u=0
$$

(sometimes called $(k)$-monogenic or polymonogenic functions) given in [5] (see also [6]) and further the classical Almansi theorem [7] for polyharmonic functions.

Supposing that there exists a representation of the solutions of $D v=0$ by means of certain differential operators as it was discussed e.g. in the forthcoming article we prove a close connection between the solutions of (1) and the solutions of (2). 


\section{An Almansi-type decomposition}

Proposition 1. For any real valued, continuously differentiable function $\varphi\left(x_{0}\right)$ of the variable $x_{0}$ and for each function $\tilde{w} \in C^{1}\left(\Omega, C l_{p, q}\right)$ we have

(i) $\partial\left(\varphi\left(x_{0}\right) \tilde{w}\right)=\varphi^{\prime}\left(x_{0}\right) \tilde{w}+\varphi\left(x_{0}\right)(\partial \tilde{w})$

(ii) $D\left(\varphi\left(x_{0}\right) \tilde{w}\right)=\varphi^{\prime}\left(x_{0}\right) \tilde{w}+\varphi\left(x_{0}\right)(D \tilde{w})$

(iii) If $u \in C^{1}\left(\Omega, C l_{p, q}\right)$ is a solution of $D u=0$ then $\alpha u$ with $\alpha \in \mathbb{R}$ is a solution of $D u=0$ also.

Proof. Using the relation $\tilde{w} \varphi\left(x_{0}\right)=\varphi\left(x_{0}\right) \tilde{w}$ which is true for any real valued function $\varphi$ and all $\tilde{w} \in C\left(\Omega, C l_{p, q}\right)$ the assertions can be proved by direct calculation.

To find representations for the solutions of the iterated equation (1) we first choose a new function $w_{1}$ as

$$
w_{1}:=D^{m-1} w-\frac{1}{x_{0}} D^{m-2} w
$$

which obeys the differential equation

$$
D w_{1}+\frac{1}{x_{0}} w_{1}=0
$$

This can be proved immediately using proposition 1(ii) and equation (1). Further we define the functions

$$
w_{k}:=D^{m-k} w-\frac{k}{x_{0}} D^{m-k-1} w, \quad k=2,3, \ldots, m-1
$$

for which the relations

$$
D w_{k}+\frac{1}{x_{0}} w_{k}=w_{k-1}, \quad k=2,3, \ldots, m-1
$$

hold. With $k=m-1$ equation (4) leads to

$$
w_{m-1}=D w-\frac{m-1}{x_{0}} w
$$

Setting $w_{m}:=w$ we get from (3), (5) and (6) a system of $m$ differential equations for the functions $w_{k}, k=1, \ldots, m$. With the vector $W:=\left(w_{1}, \ldots, w_{m}\right)^{t}$ and the matrices $A=\left(a_{j k}\right)$ with

$$
a_{j k}= \begin{cases}1 / x_{0} & \text { for } \quad 1 \leq j=k \leq m-1 \\ (1-m) / x_{0} & \text { for } j=k=m \\ -1 & \text { for } j=k+1,1 \leq k \leq m-1 \\ 0 & \text { else }\end{cases}
$$


and $B=\left(b_{j k}\right)$ with

$$
b_{j k}= \begin{cases}c & \text { for } \quad 1 \leq j=k \leq m \\ 0 & \text { else }\end{cases}
$$

this system can be given in matrix notation as

$$
\partial W+A W+B \bar{W}=0
$$

Writing $\partial W$ means the application of the operator $\partial$ to each component of $W$.

Let $P=\left(p_{j k}\right)$ denote a $m \times m$-matrix the components of which are real valued, continuously differentiable functions of $x_{0}$. By the transformation $W=P U$ in connection with proposition 1(i) equation (7) leads to the relation

$$
P(\partial U)+\left(P^{\prime}+A P\right) U+P B \bar{U}=0
$$

for the new unknown vector $U=\left(u_{1}, \ldots, u_{m}\right)^{t}$.

The request $P^{\prime}+A P=0$ can be satisfied by the matrix $P$ with

$$
p_{j k}= \begin{cases}\frac{x_{0}^{j-k-1}}{(j-k) !} & \text { for } \quad 1 \leq k \leq j, 1 \leq j \leq m-1 \\ 0 & \text { for } \quad j<k \leq m, 1 \leq j \leq m-1 \\ -\frac{1}{k} \frac{x_{0}^{m-k-1}}{(m-k-1) !} & \text { for } \quad j=m, 1 \leq k \leq m-1 \\ x_{0}^{m-1} & \text { for } \quad j=k=m\end{cases}
$$

which is nonsingular since $\operatorname{det} P=1$. Thus for the vector $U$ we have the simple system

$$
\partial U+c \bar{U}=0
$$

which states that each component $u_{k}$ of the vector $U$ obeys the generalized Bers-Vekua equation

$$
D u_{k}=\partial u_{k}+c \bar{u}_{k}=0, \quad k=1, \ldots, m
$$

The focal question is which form has the function $w=w_{m}$, the $m$-th component of the vector $W$. From $W=P U$ we have

$$
w \equiv w_{m}=\sum_{k=1}^{m} p_{m k} u_{k}=\sum_{k=1}^{m-1}-\frac{1}{k} \frac{x_{0}^{m-k-1}}{(m-k-1) !} u_{k}+x_{0}^{m-1} u_{m}
$$

Finally with proposition 1 (iii) the function $w$ can be written in the form

$$
w=\sum_{k=0}^{m-1} x_{0}^{k} v_{k}
$$

where the $v_{k}$ are solutions of $D v_{k}=0$ and we have the following 
Theorem 1. 1. Let the functions $v_{k}, k=0,1, \ldots, m-1$, be solutions of the generalized Bers-Vekua equation $D v_{k}=0$ in $\Omega$. Then the function $w$ according to (8) represents a solution of the iterated generalized Bers-Vekua equation $D^{m} w=0$ in $\Omega$.

2. For each solution $w$ of (1) defined in $\Omega$ there exist solutions $v_{k}, k=0,1, \ldots, m-1$, of $D v_{k}=0$ in $\Omega$ such that $w$ can be written in the form (8).

To bring out the connection between a solution $w$ of (1) and the functions $v_{k}$ in the representation (8) we first prove the relation

$$
D^{l} w=\sum_{k=l}^{m-1} \frac{k !}{(k-l) !} x_{0}^{k-l} v_{k}, \quad l=0,1, \ldots, m-1
$$

This can be considered as a linear system of $m$ equations for the functions $v_{k}, k=0, \ldots$, $m-1$, which has the form

$$
M V=\hat{W} \quad \text { with } \quad V=\left(v_{0}, \ldots, v_{m-1}\right)^{t} \quad \text { and } \quad \hat{W}=\left(w, D w \ldots, D^{m-1} w\right)^{t}
$$

The coefficient matrix $M=\left(m_{i k}\right)$ with

$$
m_{i k}= \begin{cases}\frac{(k-1) !}{(k-i) !} x_{0}^{k-i} & \text { for } i \leq k \\ 0 & \text { for } i>k\end{cases}
$$

is non singular, its inverse $M^{-1}=\left(\mu_{i k}\right)$ is given by

$$
\mu_{i k}= \begin{cases}\frac{(-1)^{i+k}}{(i-1) !(k-i) !} x_{0}^{k-i} & \text { for } i \leq k \\ 0 & \text { for } i>k\end{cases}
$$

Thus the solution of system (9) can be written as

$$
v_{k}=\sum_{l=0}^{m-k-1} \frac{(-1)^{l}}{k ! l !} x_{0}^{l} D^{k+l} w, \quad k=0,1, \ldots, m-1
$$

and we have the

Lemma 1. For each solution $w$ of (1) in the form (8) the functions $v_{k}$ are determined uniquely by (10).

In $C l_{0,1}$ the operator $\partial$ reduces to the Cauchy-Riemann operator and eq. (1) to the iterated Bers-Vekua equation for which in [8] a corresponding representation theorem was proved. For the iterated Dirac operator $\tilde{\partial}^{k}$ with $\tilde{\partial}=\sum_{j=1}^{n} e_{j} \partial / \partial x_{j}$ a similar decomposition was proved in [9], whereas in [10] a unified approach to decomposing kernels of iterated operators was investigated. 


\section{Differential operators for the solutions}

Now let us consider the case when the solutions of $D v=0$ can be represented by means of a suitable differential operator of Bauer-type acting on solutions of $\partial u=0$. In the paper mentioned above a sufficient condition on the coefficient $c$ in $D v=0$ was given for the existence of such a differential operator with which Clifford holomorphic functions $g$ can be transformed into solutions $v$ of $D v=0$ by

$$
v=\sum_{j=0}^{N} a_{j}\left(x_{0}\right)\left(g \bar{\partial}^{j}\right)+\sum_{j=0}^{N-1} b_{j}\left(x_{0}\right)\left(\partial^{j} \bar{g}\right), N \in \mathbb{N}
$$

In particular for the coefficient $c\left(x_{0}\right)=N / x_{0}, N \in \mathbb{Z}$, such a Bauer-type differential operator exists and the coefficients $a_{k}$ and $b_{k}$ can be given in an explicite form.

Let us assume that there exists a representation for the functions $v_{k}$ in (8) of the form

$$
v_{k}=\sum_{j=0}^{N} a_{j}\left(x_{0}\right)\left(g_{k} \bar{\partial}^{j}\right)+\sum_{j=0}^{N-1} b_{j}\left(x_{0}\right)\left(\partial^{j} \bar{g}_{k}\right), k=0, \ldots, m-1
$$

with suitabel functions $g_{k}$ which are solutions of $\partial g_{k}=0, k=0, \ldots, m-1$. With the operator $\delta$ defined by

$$
\delta u:=(u \bar{\partial})-(\partial u), \delta^{j+1} u:=\delta\left(\delta^{j} u\right), \delta^{0} u:=u
$$

by direct calculation we can prove the

\section{Proposition 2.}

(i) For any Clifford holomorphic function $g_{k}$ the function $u$ according to

$$
u=\sum_{k=0}^{m-1} x_{0}^{k} g_{k}
$$

represents a solution of $\partial^{m} u=0$ (see also [5]).

(ii) For a function u given by (12) we have the relation

$$
\delta^{j} u=\sum_{k=0}^{m-1} x_{0}^{k}\left(g_{k} \bar{\partial}^{j}\right)
$$

Now from the representation (8) with the functions $v_{k}$ given in (11) and with proposition 2(ii) we get the following form for the solutions of (1)

$$
w=\sum_{j=0}^{N} a_{j}\left(x_{0}\right)\left(\delta^{j} u\right)+\sum_{j=0}^{N-1} b_{j}\left(x_{0}\right) \overline{\left(\delta^{j} u\right)}
$$

Theorem 2. In the case of the existence of Bauer-type operators for the representation of the solutions of the generalized Bers-Vekua equation $D v=0$ the solutions of the iterated generalized Bers-Vekua equation $D^{m} w=0$ can be given in terms of solutions of the iterated generalized Cauchy-Riemann equation $\partial^{m} u=0$ by a differential operator in the form (13). 


\section{References}

[1] Obolashvili E. Partial Differential Equations in Clifford Analysis. Longman: Harlow, 1998.

[2] Malonek HR. Generalizing the (F,G)-derivative in the sense of Bers. In Clifford Algebras and their Applications in Mathematical Physics, Dietrich V, Habetha K, Jank G (eds). Kluwer: Dordrecht, 1998; 247-257.

[3] Goldschmidt B. Regularity properties of generalized analytic vectors in $\mathbb{R}^{n}$. Mathematische Nachrichten 1981; 103: 245-254.

[4] Berglez P. Representation of pseudoanalytic functions in the space. In More Progresses in Analysis, Begehr H, Nicolosi F (eds). World Scientific Publ: Singapore, 2008; 1119 1126.

[5] Constales D, De Almeida R, Kraußhar RS. On Cauchy estimates and growth orders of entire solutions of iterated Dirac and generalized Cauchy-Riemann equations. Mathematical Methods in the Applied Sciences 2006; 29: 1663-1686.

[6] Brackx F. On $(k)$-monogenic functions of a quaternion variable. Function Theoretic Methods in Differential Equations. Research Notes in Mathematics, vol. 8. Pitman: London, 1976; 22-44.

[7] Almansi E. Sull' integrazione dell' equazione differenziale $\Delta^{2 n}=0$. Annali di Matematica Pura ed Applicata (Ser. 3) 1899; 2: 1-51.

[8] Berglez P. On the solutions of the iterated Bers-Vekua equation. In Functional-Analytic and Complex Methods, their Interactions, and Applications to Partial Differential Equations, Florian H. et al. (eds). World Scientific Publ: Singapore, 2001; 266-275.

[9] Malonek HR, Ren G. Almansi-type theorems in Clifford analysis. Mathematical Methods in the Applied Sciences 2002; 25: 1541-1552.

[10] Ren G, Malonek HR. Decomposing kernels of iterated operators - a unified approach. Mathematical Methods in the Applied Sciences 2007; 30: 1037-1047. 